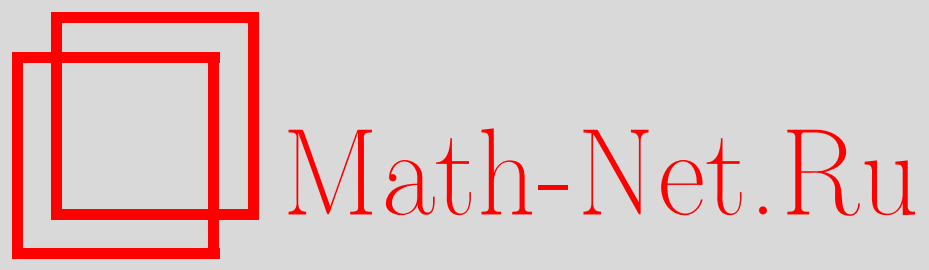

В. П. Маслов, Об одном классе лагранжевых многообразий, отвечающем вариационным задачам, задачам теории управления и термодинамики, Функи. анализ и его прил., 1998, том 32, выпуск 2, 89-91

DOI: https://doi.org/10.4213/faa415

Использование Общероссийского математического портала MathNet.Ru подразумевает, что вы прочитали и согласны с пользовательским соглашением

http://www . mathnet.ru/rus/agreement

Параметры загрузки:

IP : 54.198 .67 .100

26 апреля 2023 г., 18:20:13

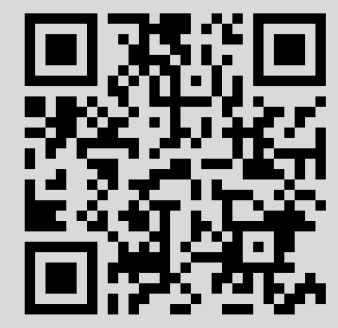




\title{
Об одном классе лагранжевых многообразий, отвечающем вариационным задачам, задачам теории управления и термодинамики
}

\author{
(c) 1998. В. П. МАСлов
}

Автором была предпринята попытка математически аксиоматизировать феноменологическую термодинамику [1]. Внимательное изучение задач термодинамики показало, что используемые в физике уравнения состояний приводят к более узкому классу лагранжевых многообразий, чем рассматривал ранее автор. Этот узкий класс важен также и для задач теории управления.

1. Класс канонических преобразований. В задачах теории управления и вариационных задачах минимизируется обычно функционал, определяемый неотрицательным лагранжианом $L(\dot{x}, x)$. Он порождает каноническое преобразование, отвечающее соответствующему гамильтониану $H(p, x)$, $p \in \mathbb{R}^{N}, x \in \mathbb{R}^{N}$. Соответствие $L(\dot{x}, x) \leftrightarrow H(p, x)$ предполагается взаимно однозначным, т. е. система $\dot{x}=H_{p}(p, x)$ однозначно разрешима относительно $p$ при всех $x$. Каждый гамильтониан указанного вида порождает полугруппу канонических преобразований.

Рассмотрим каноническое преобразование $K$ указанного вида, которое отображает лагранжеву плоскость $x=x_{0}$ в $\mathbb{R}^{2 N}$, параллельную $p$ и обозначаемую через $A_{x_{0}}$, в лагранжево многообразие $\Lambda_{x_{0}} \in \mathbb{R}^{2 N}$, диффеоморфно проектирующееся на $x$-плоскость при любом $x_{0} \in \mathbb{R}^{N}$. Класс таких преобразований обозначим через $\mathscr{K}$.

Пусть $\mathscr{S}$ - полугруппа, порожденная всеми такими каноническими преобразованиями.

2. Класс лагранжевых многообразий. Обозначим через $\mathscr{L}$ класс лагранжевых многообразий вида $\Lambda=K A_{x_{0}}$ для всех $x_{0}$ и всех $K \in \mathscr{S}$. Понятия существенной и несущественной точек лагранжева многообразия определены в [1]. Это определение фактически продублировано ниже (в доказательстве следующей теоремы 1).

ТЕорема 1. Каноническое преобразование $K \in \mathscr{S}$ переводит несуиественные точки лагранжева многообразия $\Lambda \in \mathscr{L}$ в несущественнье точки лагранжева многообразия $K \Lambda$.

Наметим доказательство. Пусть сначала $K=K_{1} \cdot K_{2}$, где $K_{1}, K_{2} \in \mathscr{K}$, $\Lambda=K_{2} A_{x_{0}}$, а $\Lambda_{1}=K_{1} \Lambda$. Пусть точке $x_{1}$ на координатной плоскости в фазовом пространстве $\mathbb{R}^{2 N}$ отвечают точки $\alpha_{1}\left(x_{1}\right), \ldots, \alpha_{k}\left(x_{1}\right)$ на $\Lambda_{1}$, которые проектируются в точку $x_{1}$.

Пусть $S(\alpha)=\int p d x$ - действие на $\Lambda_{1}$, а $\alpha_{i_{0}}\left(x_{1}\right)$ - существенная точка, т. е. по определению $S\left(\alpha_{i_{0}}\left(x_{1}\right)\right)=\min _{i} S\left(\alpha_{i}\left(x_{1}\right)\right)$. Обозначим через $S_{1}\left(x, x_{0}\right)$,

* Работа выполнена при финансовой поддержке ИНТАС и РФФИ, совместный грант 95-91. 
$S_{2}\left(x, x_{0}\right)$ производящие функции (действия), отвечающие $K_{1}$ и $K_{2}$ соответственно.

Поскольку $\Lambda_{1}=K_{1} K_{2} A_{x_{0}}$,

$$
S\left(\alpha_{i}\left(x_{1}\right)\right)=S_{1}\left(x_{1}, \xi_{i}\right)+S_{2}\left(\xi_{i}, x_{0}\right),
$$

где $\xi_{i}$ такова, что

$$
\frac{\partial S_{1}}{\partial \xi_{i}}\left(x_{1}, \xi_{i}\right)=-\frac{\partial S_{2}}{\partial \xi_{i}}\left(\xi_{i}, x_{0}\right)
$$

Следовательно,

$$
S\left(\alpha_{i_{0}}\left(x_{1}\right)\right)=\min _{i}\left(S_{1}\left(x_{1}, \xi_{i}\right)+S_{2}\left(\xi_{i}, x_{0}\right)\right) .
$$

Пусть $K_{3} \in \mathscr{K}$ и $S_{3}\left(x, x_{0}\right)$ - производящая функция преобразования $\mathscr{K}$. Рассмотрим $\Lambda_{2}=K_{3} \Lambda_{1}$. Пусть $\alpha^{\prime}\left(x_{1}\right)$ - сушественная точка на $\Lambda_{2}$, проектирующаяся в точку $x_{1} \in \mathbb{R}^{N}$ и $S^{\prime}(\alpha)=\int p d x$ на $\Lambda_{2}$; тогда

$$
S^{\prime}\left(\alpha^{\prime}\left(x_{1}\right)\right)=\min _{i} S^{\prime}\left(\alpha_{i}^{\prime}\left(x_{1}\right)\right) \text {, }
$$

где $\alpha_{i}^{\prime}\left(x_{1}\right)$ - все точки на $\Lambda_{2}$, которые проектируются в $x_{1}$.

Аналогично предыдушему

$$
S^{\prime}\left(\alpha_{i}^{\prime}\left(x_{1}\right)\right)=\left\{S_{3}\left(x_{1}, \xi_{i}\right)+S_{2}\left(\xi_{i}, \eta_{i}\right)+S_{1}\left(\eta_{i}, x_{0}\right)\right\},
$$

где $\xi_{i}, \eta_{i}$ удовлетворяют соотношениям

$$
\frac{\partial S_{3}}{\partial \xi}\left(x_{1}, \xi\right)=-\frac{\partial S_{2}}{\partial \xi}(\xi, \eta), \quad \frac{\partial S_{2}}{\partial \eta}(\xi, \eta)=-\frac{\partial S_{1}}{\partial \eta}\left(\eta, x_{0}\right),
$$

а значит,

$$
\begin{aligned}
S^{\prime}\left(\alpha^{\prime}\left(x_{1}\right)\right)=\min _{i} S^{\prime}\left(\alpha_{i}^{\prime}\left(x_{i}\right)\right) & =\min _{i}\left(S_{1}\left(x_{1}, \xi_{i}\right)+S_{2}\left(\xi_{i}, \eta_{i}\right)+S\left(\eta_{i}, x_{0}\right)\right) \\
& =\min _{i}\left\{S\left(x_{1}, \xi_{i}\right)+\min _{j}\left(S_{2}\left(\xi_{i}, \eta_{j}\right)+S_{1}\left(\eta_{j}, x\right)\right)\right\} \\
& =\min _{i}\left(S_{3}\left(x, \xi_{i}\right)+S\left(\alpha_{j_{0}}\left(\xi_{j}\right)\right)\right) .
\end{aligned}
$$

Следовательно, в определении точки $\alpha^{\prime}\left(x_{1}\right)$ участвуют только существенные точки $\alpha_{j_{0}}\left(\xi_{i}\right)$ многообразия $\Lambda_{1}$.

Продолжая рассуждения рекуррентным образом, получим утверждение теоремы.

2. Термодинамика. Перейдем к термодинамике. Пусть заданы $N$ экстенсивных (объем, энтропия и т.д.) и $N$ сопряженных им интенсивных (давление, температура и т.д.) термодинамических величин. Рассмотрим фазовое пространство $\mathbb{R}^{2 N}$, где интенсивные величины - координаты, а экстенсивные - импульсы. Пусть, как обычно, задано $N$ соотношений между ними (уравнения состояний).

ТЕОРемА 2. Если уравнения состояний определяют лагранжево многообразие из класса $\mathscr{L}$, то первый и второй законы термодинамики будут выполнень. 
ГипотезА. Туннельный канонический оператор [2] на $\Lambda \in \mathscr{L}$, где роль малого параметра играет $h=k T / N(k-$ постолнная Больимана, $T$ температура, $N$ - иисло частии), определяет асимптотику большой статистической суммь, если $V / N \rightarrow$ const, где $V$ - объем статистической cистемы.

Для широкого класса задач (например, в приближении так называемого среднего поля) эта гипотеза доказана автором и его учениками.

Точки фазового перехода в этой концепции интерпретируются как каустики и фокальные точки, лежащие в области существенных точек. Поэтому более детальная асимптотика туннельного канонического оператора в духе теории катастроф В. И. Арнольда освещает асимптотическое поведение системы вблизи критических точек фазовых переходов первого и второго рода.

ЗАмечАниЕ. Подобное же геометрическое «квантование» может быть осуществлено и для уравнений Беллмана, в частности, когда они описывают задачу о больших уклонениях (см., например, [3]).

\section{ЛитеРАТУРА}

1. Маслов В. П. Функц. анализ и его прил., 28, вып. 4, 28-41 (1994). 2. Маслов B. П. Асимптотические методы и теория возмущений. Наука, М., 1988. 3. Del Moral P. Maslov Optimization Theory: Topological Aspects. In: Idempotency. Publ. Inst. Newton (J. Gunawardena, ed.), Cambridge University Press, 1998, pp. 354-391.

Московский государственный университет, им. М. В. Ломоносова, физический факультет e-mail: maslov@ipmnet.ru

Поступило в редакцию 2 марта 1998 г.

\section{Новая аффинная $M$-секстика}

(C) 1998. С. Ю. Оревков

Аффинной $M$-кривой мы будем называть аффинную вещественную алгебраическую кривую $C$, имеющую максимально возможное число $\left(\mathrm{m}^{2}-\right.$ $m+2) / 2$ компонент связности, где $m-$ степень кривой $C$. Это эквивалентно тому, что проективное замыкание $\bar{C}$ кривой $C$ является проективной $M$-кривой, т. е. имеет максимально возможное число $1+(m-1)(m-2) / 2$ компонент связности и пересекает бесконечно удаленную прямую $L$ в $m$ различных вещественных точках, лежащих на одной компоненте связности кривой $C$. Это определение отличается от приведенного в $[1,3]$, однако нам оно представляется более естественным.

В работе [1] построено 33 изотопических типа $M$-кривых степени 6 . Другие построения (причем более подробно изложенные) этих 33 кривых приведены в $[2]$. В $[1,3]$ объявлено также, что все остальные изотопические типы, кроме 9 , нереализуемы, однако, доказательства по крайней мере трех из этих 